\title{
Optical coherence tomographic pattern and focal electroretinogram in patients with retinitis pigmentosa
}

A Oishi, H Nakamura, I Tatsumi, M Sasahara, H Kojima, M Kurimoto, A Otani and N Yoshimura

\section{Abstract}

Purpose The foveal function of patients with retinitis pigmentosa (RP) has been estimated by visual acuity (VA) or visual field (VF) tests. In the present study, the potential of optical coherence tomography (OCT) and focal electroretinogram (fERG) for monitoring macular function in RP patients was investigated.

Design Cross-sectional observational study. Methods A total of 56 eyes of 56 patients with RP underwent ophthalmic examination including VA, VF, fERG, and OCT. Patients were morphologically divided into three groups by the appearance of photoreceptor inner/outer segment junction (IS/OS) that were depicted with OCT; type 1: no IS/OS visible, type 2: IS/OS was visible but the length was $\leqslant 2 \mathrm{~mm}$, and type 3 : IS/OS $>2 \mathrm{~mm}$ was confirmed. Functional results for VA and fERG were compared and analysed based on the three groups.

Results The average VA of type 1 patients was significantly lower than that of types 2 or 3 patients $(P<0.001)$. There were no significant VA differences detected between types 2 and 3 patients. While most of the type 1 patients (21/ 22) showed non-recordable fERG, 3 out of 18 type 2 patients and none of type 3 patients showed non-recordable fERG. Significant differences of the fERG amplitudes were observed among the three groups (a-wave, $b$-wave, and $O P, P<0.001$ in all three components). However, the implicit time showed no difference between type 2 and 3 . Conclusions Analysing the IS/OS with OCT and the amplitudes of fERG may be helpful for monitoring RP patients in addition to VA and VF. Eye (2009) 23, 299-303; doi:10.1038/sj.eye.6703077; published online 14 March 2008
Keywords: retinitis pigmentosa;

electroretinography; optical coherence tomography; photoreceptor; maculae

\section{Introduction}

Retinitis pigmentosa (RP) is a heterogeneous group of hereditary diseases that affects photoreceptors and the retinal pigment epithelium. As most of the mutations lie in genes expressed in rod photoreceptors, the disease typically starts with island scotoma in the midperipheral visual field, and progresses to ring-shaped or concentric scotoma. In the very small central visual field left in advanced cases, some retain good function throughout the lifetime and others begin to degenerate, resulting in total blindness. Accurate and detailed measurement of the remaining function of cones or central macula is helpful not only for monitoring the progression of RP but also for future therapies.

There are several ways of monitoring macular function, including visual acuity (VA), contrast sensitivity, color vision, statistic visual field and electroretinogram (ERG). ERG is an objective measurement of retinal function that has been used for diagnosis and severity of the disease for many years. In contrast to conventional ERG, focal ERG (fERG) and multifocal ERG (mfERG) stimulate a small part of the retina and record the response from the area; these methods have advantage of monitoring macular function. The benefit of $\mathrm{fERG}^{1-3}$ and $\mathrm{mf} \mathrm{ERG}^{4-6}$ in monitoring RP patients has already been reported.

Optical coherence tomography (OCT) is unique as it provides in vivo morphological information and has been used in many retinal
Department of

Ophthalmology and Visual Sciences, Kyoto University Graduate School of Medicine, Kyoto, Japan

Correspondence:

H Nakamura,

Department of

Ophthalmology and Visual Sciences,

Kyoto University Graduate School of Medicine, 54 Shougoin Kawahara-cho, Sakyo-ku,

Kyoto 606-8507, Japan

Tel: + 81757513248 ;

Fax: +81757520933

E-mail: hajime88@

kuhp.kyoto-u.ac.jp

Received: 6 July 2007

Accepted in revised form: 5 November 2007; Published online: 14 March 2008

Part of the content was presented at ARVO 2007 annual meeting, Fort Lauderdale, FL, USA Conflict of interest: None 
disease, for example, macular oedema/thickening in various disease ${ }^{7}$ or retinal nerve fibre layer thinning in glaucoma. ${ }^{8}$ These studies coincidentally showed the close relationship between retinal function and its morphology. Some authors also studied OCT and mfERG and showed a correlation between mfERG amplitude and retinal thickness. ${ }^{9-12}$ Since the time when OCT was used mainly to measure thickness of specific layer, the resolution of the image has been dramatically improved, and the device now can clearly recognize specific structure of the retina. Among the retinal structures observed with the latest model of OCT, the status of photoreceptor inner/outer segment junction (IS/OS), which is depicted as a high-intensity band just above the retinal pigment epithelium, ${ }^{8}$ is emerging as a useful parameter for monitoring macular morphology and function..$^{13-15}$ Sandberg et al ${ }^{16}$ examined the retinal thickness and IS/OS in RP patients and reported that abnormal thickening or thinning of the retina and the absence of IS/OS are correlated with low VA. Similar results were also reported in Stargardt disease. ${ }^{17}$

OCT may provide practical, noninvasive, and less time-intensive means to monitor macular function of RP patients. In the present study, we examined whether OCT and fERG have any advantages as objective examinations in monitoring the progression of RP.

\section{Materials and methods}

\section{Subjects}

The subjects of this study included patients with RP who visited the Department of Ophthalmology, Kyoto University Hospital between November 2006 and April 2007. One eye of each patient was randomly included in the study. RP was diagnosed with the presence of night blindness, characteristic fundus appearance, concentric, ring-shaped or island scotoma corresponding to the fundus appearance, and nonrecordable or subnormal conventional ERG; conventional ERG was recorded according to the International Society for Clinical Electrophysiology of Vision (ISCEV) recommended in 2004. The patients with best-corrected VA worse than 0.1 $(20 / 200)$, with myopia $<-6 \mathrm{D}$, or with macular disease (eg, macular hole, epiretinal membrane or cystoid macular oedema) were excluded from the study.

The patients underwent an ophthalmic examination including best-corrected VA measurement, Goldmann perimetry, measurements with 3D OCT-1000 or its prototype Fourier domain OCT (Topcon, Tokyo, Japan) and fERG (Mayo corp., Inazawa, Japan). The examinations were performed within 3-month intervals. Six-millimeter horizontal macular scan was performed with OCT after patients' pupils were dilated. The obtained images were corrected for axial motion with standard algorithms of the manufacturer. These images were analysed by one of the authors (AO), who was masked from other examination results, and the patients were classified into three groups with the status of IS/ OS. The classification was as follows: type 1, no IS/OS detected; type 2, IS/OS was depicted with OCT but the length was $2 \mathrm{~mm}$ of IS/OS was confirmed (Figure 1).

fERG was performed under the following setting: fERG was elicited by $15^{\circ}$ stimulus positioned on the fovea and monitored by an infrared fundus camera. The luminance of white stimulus light and background illumination were 181.5 and $6.9 \mathrm{~cd} / \mathrm{m}^{2}$, respectively. A background field of $45^{\circ}$ visual angle was projected to the eye from the fundus camera. The Burian-Allen bipolar contact lens electrode was used for the fERG recordings. This electrode allowed not only low noise recordings but also permitted a clear view of the fundus that was displayed on a television monitor. The left ear served as a ground.

The fERGs were recorded with 5-Hz rectangular stimuli (100 ms light on and $100 \mathrm{~ms}$ light off) after patients' pupils were dilated. The stimulus spot was centred on the fovea. A total of 500 responses were averaged by a signal processor Neuropack MEB-2204 (Nihon Kohden, Tokyo, Japan). The fERG response was digitized at $10 \mathrm{kHz}$ with a band-pass filter of $5-500 \mathrm{~Hz}$ for the a- and b-waves, and $50-500 \mathrm{~Hz}$ for OPs. The amplitudes and implicit times of the a- and b-waves, and the mean amplitudes and implicit times of the first three oscillatory potentials (OP1-OP3) were compared between the three types. The amplitude of the a-wave was measured from the baseline to the peak of the

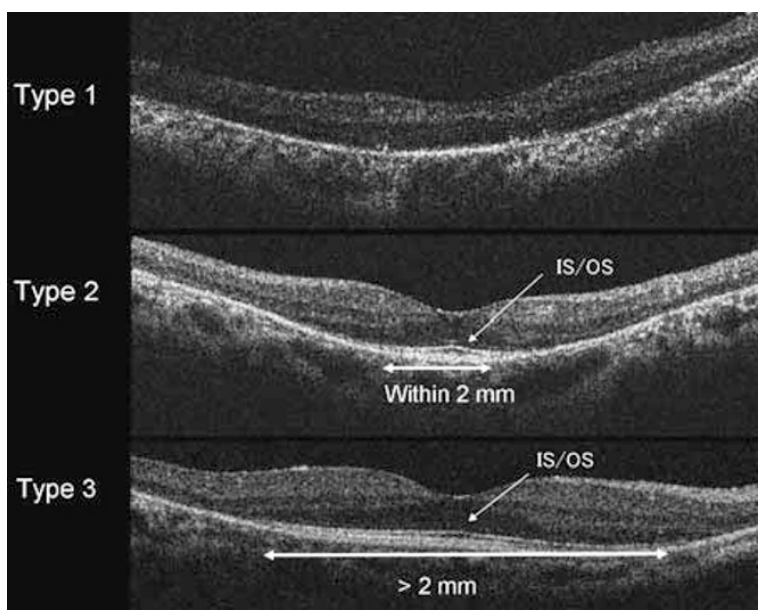

Figure 1 Examples of classification used in the study. Type 1: photoreceptor inner segment/outer segment junction (IS/OS) was completely absent. Type 2: IS/OS is depicted with OCT but the length was less than $2 \mathrm{~mm}$. Type 3: More than $2 \mathrm{~mm}$ of IS/OS was confirmed. 
a-wave. The amplitude of the b-wave was measured from the trough of the a-wave to the peak of the b-wave. The amplitude of each OP wavelet was measured from a baseline to its peak. Results of the fERG responses were normalized by normal values obtained from age-matched 41 normal subjects.

The study always observed the tenets of the Declaration of Helsinki. Informed consent was obtained from each patient after full explanation of the examinations. We certify that all applicable institutional and governmental regulations concerning the ethical use of human volunteers were followed during this research.

\section{Statistical analysis}

The statistical program SPSS was used to analyse the sample data. The differences between each group were tested with one-way ANOVA followed by Bonferroni test. For the analysis of ERG implicit time, unpaired $t$-test was used since the majority of type 1 patients had nonrecordable ERG and implicit time could not be determined.

\section{Results}

The study sample consisted of 56 eyes of 56 patients, of whom 21 were men and 35 were women. IS/OS was not visible in 22 patients who were classified as type 1 . All of the type 2 or 3 patients had IS/OS with the centre on fovea, and there was no single case with remaining IS/OS in peripheral macula but not in fovea. The demographics of the participants are shown in Table 1. There were no significant differences for average age (type 1: $46.6 \pm 17.4$, type 2: $49.6 \pm 12.5$, type $3: 52.4 \pm 17.1$, years) or refractive error (type $1:-1.7+2.3$, type 2 :

$-1.8+2.0$, and type $3:-0.8+2.9 \mathrm{D})$ between each group (Table 1).

After every patient was successfully assigned to any one of each group, average logMAR of each group was compared; type 1 patients $(0.4 \pm 0.3)$ had worse logMAR compared with type $2(0.0 \pm 0.2)$ or $3(0.0 \pm 0.2)$. The difference between types 2 and 3 was not statistically significant (Figure 2).
Then, we analysed the fERG of each group. Almost all the patients classified as type 1 presented nonrecordable fERG $(21 / 22)$, while only 3 patients out of 18 did so in type 2 , and all patients were recordable in type 3 (Figure 3 ). The average $b / a$ ratio were as follows: type 1 , 4.3 (one case); type 2, $2.7 \pm 0.7$; and type 3, $2.5 \pm 0.8$, respectively. There was no negative fERG pattern in the examined patients. When comparing the normalized amplitude of a- and b-waves and oscillatory potential $(\mathrm{OP})$, all waves showed progressive decline with the definition $(P<0.001)$. The exact values of relative percentage to normal control were as follows: type 1 ,

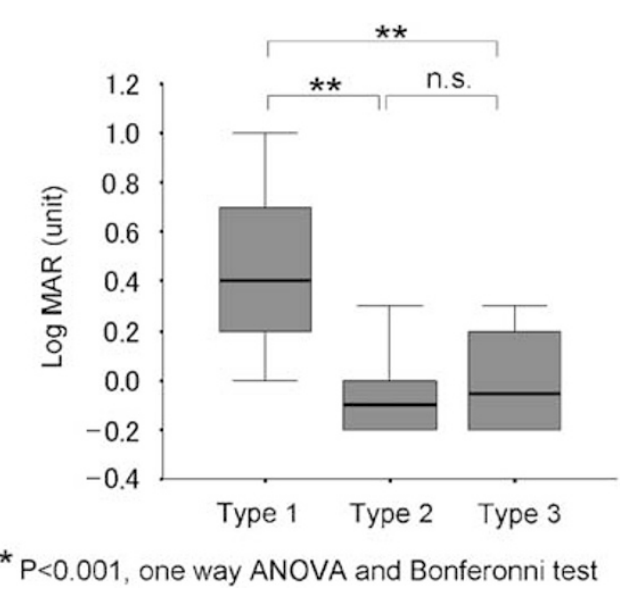

Figure 2 LogMAR of each group. Type 1 had significantly lower $\log$ MAR compared with the other two groups $(P<0.001)$. There was no significant difference between types 2 and 3 .

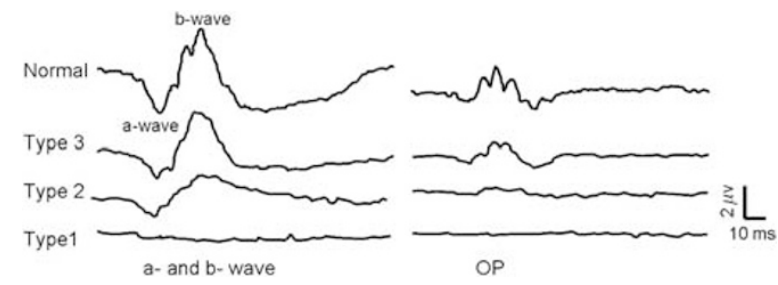

Figure 3 Representative fERG results of each group, left: a- and b-wave, right: OP. While type 3 patients showed almost normal fERG, almost all the type 1 patients were nonrecordable.

Table 1 Demographics of patients in IS/OS subgroup

\begin{tabular}{lccc}
\hline & Type 1 & Type 2 & Type 3 \\
\hline Age (years) & $46.6 \pm 17.4$ & $49.6 \pm 12.5$ & $52.4 \pm 17.1$ \\
Sex (male/female, eyes) & $(8 / 14)$ & $(8 / 10)$ & $(5 / 11)$ \\
Laterality (right/left, eyes) & $(9 / 13)$ & $(8 / 10)$ & $(9 / 7)$ \\
Inheritance trait (autosomal dominant/autosomal recessive/X-linked/sporadic) & $(4 / 2 / 0 / 16)$ & $(3 / 2 / 0 / 13)$ & $(3 / 2 / 0 / 11)$ \\
LogMAR (unit) & $0.4 \pm 0.3$ & $0.0 \pm 0.2$ & $0.0 \pm 0.2$ \\
Refractive error (diopter) & $-1.7 \pm 2.3$ & $-1.8 \pm 2.0$ & $-0.8 \pm 2.9$ \\
Nonrecordable fERG (eyes) & 21 & 3 & 0 \\
\hline
\end{tabular}


a-wave $1.1 \pm 5.3$, b-wave $1.7 \pm 8.2$, and OP $2.3 \pm 10.7$; type 2, a-wave $42.2 \pm 29.1$, b-wave $33.8 \pm 25.5$, and OP $34.2 \pm 27.1$; type 3, a-wave $81.1 \pm 24.7$, b-wave: $73.7 \pm 23.5$, and OP: $80.5 \pm 25.8$ (Figure 4 left). However, the implicit time of each wave was not significantly different between types 2 and 3 (a-wave: $109.5 \pm 12.6$ vs $102.8 \pm 9.0$, $P=0.074$; b-wave: $103.2 \pm 5.0$ vs $100.3 \pm 2.5, P=0.191$; OP $104.9 \pm 7.6$ vs $103.7 \pm 6.4, P=0.523$ ) (Figure 4 right).

\section{Discussion}

Examinations that detect the subjective visual function are important, because these results directly correlate with the patient symptoms. VA and VF testing are the most widely used subjective measurements in clinical practice, and most clinical trials for eye diseases utilize these tests as major valuation bases. On the other hand, difficulties in clinical trials have been pointed out in that these measures of retinal function have inter-visit variability, and small changes can be overlooked in slowly progressive diseases such as RP. ${ }^{18}$ As cone photoreceptors can be lost in the fovea before having a reduction in $\mathrm{VA}^{19}$ and the neural degeneration is irreversible, more sensitive strategy or examination in detecting RP progression is necessary. In the present study, we evaluated the utility of the objective visual examination, OCT and $\mathrm{fERG}$, as indicators of macular function in RP patients. We demonstrated that the evaluation of IS/OS depicted by OCT and measurement of cone function by fERG can be utilized as a strategy in judging the progression of RP.
IS/OS depicted with OCT is considered to represent the photoreceptor inner and outer segment junction. ${ }^{8}$ Distortion, shortening, or absence of IS/OS may indicate pathologic changes in photoreceptors as shown in $\mathrm{RP}^{16}$ as well as in macular hole, ${ }^{20}$ retinal detachment, ${ }^{14}$ retinal vein occlusion, ${ }^{21}$ central serous chorioretinopathy, ${ }^{13,15,22}$ or multiple evanescent white dot syndrome. ${ }^{23}$ Also in our patients, complete disappearance of IS/OS detected by OCT was correlated with decrease in VA, as expected. This supports the notion that morphological changes detected by OCT can reflect the function of fovea, also in RP patients. Shortening of IS/OS defined with a criteria more or less than $2 \mathrm{~mm}$, which is based on the diameter of anatomical fovea $1.85 \mathrm{~mm}^{24}$ did not correlated with the VA decrease. This result may be reasonable as VA may represent the foveal function and both type 2 and 3 groups have IS/OS in fovea. In contrast, fERG detected the difference of the partial IS/OS shortening. The fERG amplitude was significantly different between the three IS/OS groups and of note, almost all the patients with absent IS/OS (21/22) showed nonrecordable fERG. Because fERG measures the $15^{\circ}$ of macular area, the amplitude detected by fERG can represent the total macular function, which does not necessarily match with VA. ${ }^{25}$ OCT scans $6 \mathrm{~mm}$ of retina, which is approximately $20^{\circ}$, therefore, the shortening or absence of IS/OS is directly reflected as the decline in fERG amplitude.

In contrast to the amplitude, the implicit time of fERG of each group was not statistically different. When the photoreceptors degenerate, implicit time generally is elongated. ${ }^{26}$ The dissociation seen in the studied population may be due to the small sample size, or
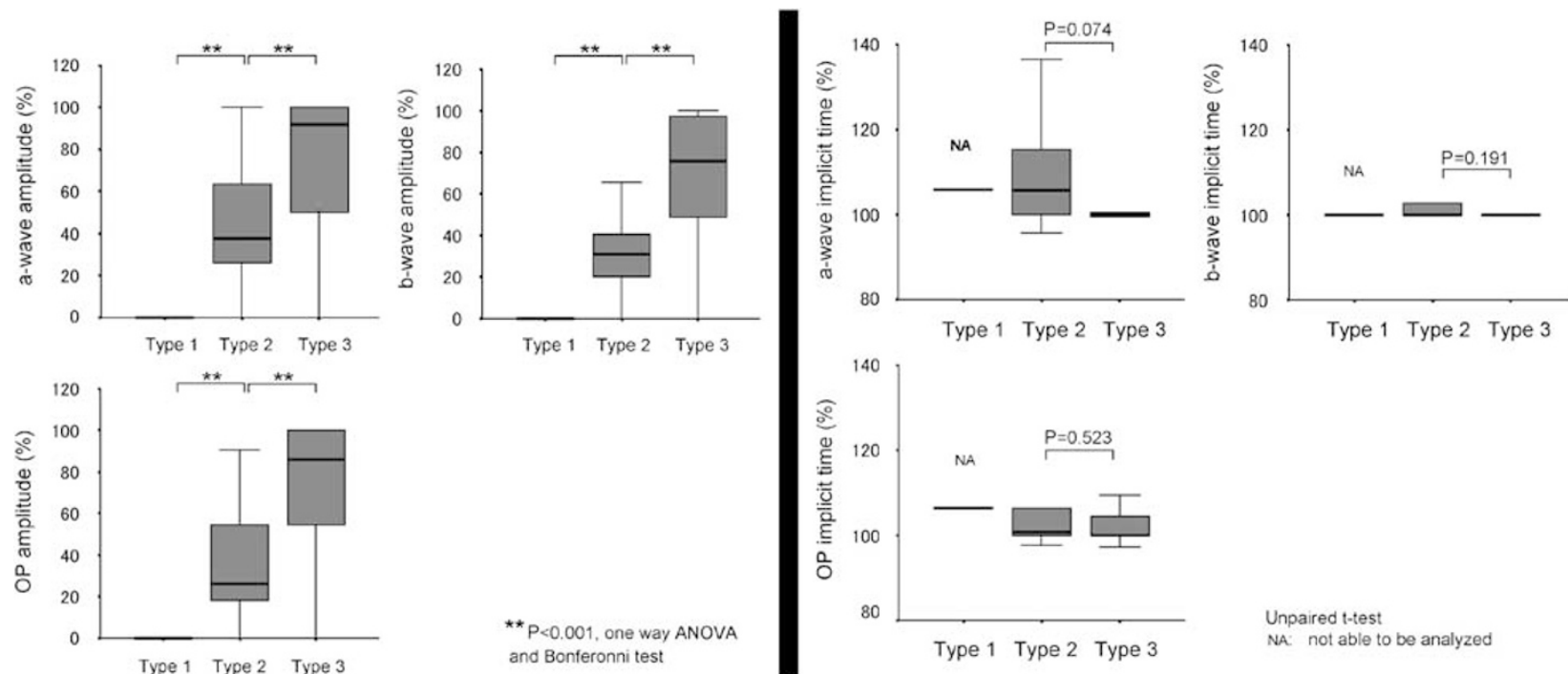

Figure 4 Left: The amplitude of fERG relative to the normal control (\%) in each group. The amplitude of a-wave, b-wave, and OP declined in accordance with the definition with IS/OS. Right: The implicit time of fERG relative to the normal control (\%) in each group. The result of type 1 could not be analysed since 21 patients out of 22 showed nonrecordable fERG. The difference between types 2 and 3 was not significant. 
perhaps indicates that the photoreceptors are decreased in number, but the remaining cells function almost normally in the disease. Further study is needed to provide a precise answer to the process of cone degeneration in the disease.

This study was cross-sectional and has limitation in providing time course information. Absence or shortening of IS/OS observed with OCT was correlated with macular function, but we cannot tell whether it was the consequence of the disease progression or the phenotypic variation of the disease. In addition, we cannot predict which patient retains good macular function and which patient loses. Further study will be necessary to clarify this issue.

Finally, we showed that the status of IS/OS depicted with OCT is correlated well with VA and amplitude of fERG and that fERG detected the difference between types 2 and 3 patients who did not exhibit significant difference in VA. These data suggest that analysing the status of IS/OS with OCT may be a good strategy that represents both morphological and functional changes in RP. And fERG is another indicator of macular function for the patients without severe VA loss.

\section{References}

1 Seiple WH, Siegel IM, Carr RE, Mayron C. Evaluating macular function using the focal ERG. Invest Ophthalmol Vis Sci 1986; 27: 1123-1130.

2 Falsini B, Iarossi G, Porciatti V, Merendino E, Fadda A, Cermola $S$ et al. Postreceptoral contribution to macular dysfunction in retinitis pigmentosa. Invest Ophthalmol Vis Sci 1994; 35: 4282-4290.

3 Ikenoya K, Kondo M, Piao C-H, Kachi S, Miyake Y, Terasaki $\mathrm{H}$. Preservation of macular oscillatory potentials in eyes of patients with retinitis pigmentosa and normal visual acuity. Invest Ophthalmol Vis Sci 2007; 48: 3312-3317.

4 Granse L, Ponjavic V, Andreasson S. Full-field ERG, multifocal ERG and multifocal VEP in patients with retinitis pigmentosa and residual central visual fields. Acta Ophthalmol Scand 2004; 82: 701-706.

5 Greenstein VC, Holopigian K, Seiple W, Carr RE, Hood DC. Atypical multifocal ERG responses in patients with diseases affecting the photoreceptors. Vision Res 2004; 44: 2867-2874.

6 Gerth C, Wright T, Heon E, Westall CA. Assessment of central retinal function in patients with advanced retinitis pigmentosa. Invest Ophthalmol Vis Sci 2007; 48: 1312-1318.

7 Mirza RG, Johnson MW, Jampol LM. Optical coherence tomography use in evaluation of the vitreoretinal interface: a review. Surv Ophthalmol 2007; 52: 397-421.

8 Costa RA, Skaf M, Melo Jr LA, Calucci D, Cardillo JA, Castro JC et al. Retinal assessment using optical coherence tomography. Prog Retin Eye Res 2006; 25: 325-353.

9 Niwa T, Terasaki H, Kondo M, Piao CH, Suzuki T, Miyake Y. Function and morphology of macula before and after removal of idiopathic epiretinal membrane. Invest Ophthalmol Vis Sci 2003; 44: 1652-1656.

10 Suzuki T, Terasaki H, Niwa T, Mori M, Kondo M, Miyake Y. Optical coherence tomography and focal macular electroretinogram in eyes with epiretinal membrane and macular pseudohole. Am J Ophthalmol 2003; 136: 62-67.

11 Terasaki H, Kojima T, Niwa H, Piao CH, Ueno S, Kondo M et al. Changes in focal macular electroretinograms and foveal thickness after vitrectomy for diabetic macular edema. Invest Ophthalmol Vis Sci 2003; 44: 4465-4472.

12 Apushkin MA, Fishman GA, Alexander KR, Shahidi M. Retinal thickness and visual thresholds measured in patients with retinitis pigmentosa. Retina 2007; 27: 349-357.

13 Eandi CM, Chung JE, Cardillo-Piccolino F, Spaide RF. Optical coherence tomography in unilateral resolved central serous chorioretinopathy. Retina 2005; 25: 417-421.

14 Schocket LS, Witkin AJ, Fujimoto JG, Ko TH, Schuman JS, Rogers $\mathrm{AH}$ et al. Ultrahigh-resolution optical coherence tomography in patients with decreased visual acuity after retinal detachment repair. Ophthalmology 2006; 113: 666-672.

15 Ojima Y, Hangai M, Sasahara M, Gotoh N, Inoue R, Yasuno $\mathrm{Y}$ et al. Three-dimensional imaging of the foveal photoreceptor layer in central serous chorioretinopathy using high-speed optical coherence tomography. Ophthalmology 2007; 114: 2197-2207.

16 Sandberg MA, Brockhurst RJ, Gaudio AR, Berson EL. The association between visual acuity and central retinal thickness in retinitis pigmentosa. Invest Ophthalmol Vis Sci 2005; 46: 3349-3354.

17 Ergun E, Hermann B, Wirtitsch M, Unterhuber A, Ko TH, Sattmann $\mathrm{H}$ et al. Assessment of central visual function in Stargardt's disease/fundus flavimaculatus with ultrahighresolution optical coherence tomography. Invest Ophthalmol Vis Sci 2005; 46: 310-316.

18 Hartong DT, Berson EL, Dryja TP. Retinitis pigmentosa. Lancet 2006; 368: 1795-1809.

19 van Meel GJ, van Norren D. Foveal densitometry in retinitis pigmentosa. Invest Ophthalmol Vis Sci 1983; 24: 1123-1130.

20 Scholda C, Wirtitsch M, Hermann B, Unterhuber A, Ergun $\mathrm{E}$, Sattmann $\mathrm{H}$ et al. Ultrahigh resolution optical coherence tomography of macular holes. Retina 2006; 26: 1034-1041.

21 Ota M, Tsujikawa A, Murakami T, Kita M, Miyamoto K, Sakamoto A et al. Association between integrity of foveal photoreceptor layer and visual acuity in branch retinal vein occlusion. Br J Ophthalmol 2007; 91: 1644-1649.

22 Piccolino FC, de la Longrais RR, Ravera G, Eandi CM, Ventre L, Abdollahi A et al. The foveal photoreceptor layer and visual acuity loss in central serous chorioretinopathy. Am J Ophthalmol 2005; 139: 87-99.

23 Nguyen MH, Witkin AJ, Reichel E, Ko TH, Fujimoto JG, Schuman JS et al. Microstructural abnormalities in MEWDS demonstrated by ultrahigh resolution optical coherence tomography. Retina 2007; 27: 414-418.

24 Hogan MJ, Alvarado JA, Weddell JE. Histology of the Human Eye: an Atlas and Textbook. Saunders: Philadelphia, 1971.

25 Biersdorf WR. The clinical utility of the foveal electroretinogram: a review. Doc Ophthalmol 1989; 73 313-325.

26 Berson EL. Electroretinographic findings in retinitis pigmentosa. Jpn J Ophthalmol 1987; 31: 327-348. 